\title{
Multi-scale contrast and relative motion-based key frame extraction
}

\author{
Naveed Ejaz' ${ }^{1}$ Sung Wook Baik², Hammad Majeed ${ }^{3}$, Hangbae Chang $^{4}$ and Irfan Mehmood ${ }^{2}$
}

\begin{abstract}
The huge amount of video data available these days requires effective management techniques for storage, indexing, and retrieval. Video summarization, a method to manage video data, provides concise versions of the videos for efficient browsing and retrieval. Key frame extraction is a form of video summarization which selects only the most salient frames from a given video. Since the automatic semantic understanding of the video contents is not possible so far, most of the existing works employ low level index features for extracting key frames. However, the usage of low level features results in loss of semantic details, thus leading to a semantic gap. In this context, the saliency-based user attention modeling technique can be used to bridge this semantic gap. In this paper, a key frame extraction scheme based on a visual attention mechanism is proposed. The proposed scheme builds static visual attention method based on multi-scale contrast instead of usual color contrast. The dynamic visual attention model is developed based on novel relative motion intensity and relative motion orientation. An efficient fusion scheme for combining three visual attention values is then proposed. A flexible technique is then used for key frame extraction. The experimental results demonstrate that the proposed mechanism provides excellent results as compared to the some of the other prominent techniques in the literature.
\end{abstract}

Keywords: Key frame extraction, Video summarization, Visual saliency, Visual attention model, Fusion mechanism, Video summary evaluation

\section{Introduction}

The amount of video data on the internet is increasing day by day primarily because of increased processing power, faster networks, cheaper storage devices, and rapid development in digital video capture and editing technologies [1]. In order to prevent the potential users of the videos from being inundated by this huge collection of videos, efficient techniques for indexing and video retrieval are needed. Video summarization is a method of generating summarized versions of the videos by extracting only the significant portions of the video [2]. The primary application of video summarization include ease in browsing and retrieval. Moreover, video summaries are also used as a pre-processing steps in many video processing

\footnotetext{
*Correspondence: irfanmehmood@ieee.org

This submission is intended for the Special Issue on Real-time Image and Video

Processing in Embedded Systems for Smart Surveillance Applications

${ }^{2}$ Department of Software, Sejong University, Seoul, South Korea

Full list of author information is available at the end of the article
}

applications. The two basic types of video summarization are key frame extraction and video skimming. The key frames or representative frames are a collection of salient frames of a given video sequence. Video skims are a collection of significantly shorter duration video segments extracted from the original video. In this paper, the focus is towards summarizing videos in the form of key frames.

Ideally, video summarization techniques must utilize the high level semantic details of the video content. However, it is currently not feasible to generally extract semantic primitives (relevant objects, actions, events) from the videos. Most of the techniques in the literature are therefore either domain specific $[3,4]$ or directly employ low level index features $[5,6]$. However, the usage of low level features is inherently associated with the loss of semantic details, thus creating a big semantic gap. In this context, some authors [7-10] use visual attention model-based schemes to bridge the semantic gap. The visual attention 
model-based approaches provide a compromise between the usage of low level features and the impractical high level features. The basic assumption in such techniques is to extract those frames as key frames which are visually important for humans based on visual attention models. In this way, the semantic details of the videos can be approximated in a better way as compared to the low level features.

In this paper, a visual attention-based mechanism for extracting key frames from the videos is proposed. The framework develops efficient visual saliency-based static and dynamic attention models and then combines them using a proposed non-linear weighted fusion mechanism. The proposed scheme has been compared with four prominent schemes in the literature [11-14]. The results demonstrate that the proposed visual attention model framework is more accurate as compared to the techniques to which it is compared.

The major contributions of this paper includes the following:

- The design of a complete and consistent framework for key frame extraction using static and dynamic visual attention clues and the non-linear fusion mechanism.

- The relative motion intensity and relative motion consistency visual attention features are proposed.

- A new non-linear scheme for integration of visual attention features is proposed.

The rest of this paper is organized as follows. Section 2 provides a brief review of the existing techniques for key frame extraction. Section 3 describes the proposed framework. The experiments and results are discussed in Section 4. Finally, Section 5 concludes the paper.

\section{Related Work}

The researchers have addressed the problem of video summarization from various perspectives. The taxonomy and review of prominent video summarization techniques can be found in $[1,2]$.

There are some classification-based supervised methods. The prominent supervised methods of video summarization includes category-specific classification for scoring importance $[15,16]$, learning to detect informative and varying subsets from videos using humangenerated summaries $[17,18]$, and learning using facets (hands, faces, objects, etc.) $[19,20]$. As it might be expected, the performance of supervised methods are heavily dependent on training data which is generally not easily available. The user annotation of the training data set may also include some bias.

The unsupervised methods mostly employ low level visual features in various strategies including clustering $([14,21]$, interest prediction [17], maximal bi-clique finding [22], and energy minimization [23]). The usage of sparse coding-based techniques have also been used for video summarization by exploiting sparsity and reconstruction error [24-27]. The global comparison-based techniques use global differences between frames to extract key frames by minimizing some objective function. Sequence reconstruction error [28, 29] and fidelity criterion [30] are the popular objective functions used. The limitation of most of the low level feature-based methods is that there is no clearly defined relationship between low level features and human perception of the salient frames, and thus, high level semantic concepts are lost, creating a semantic gap.

Ma et al. [7] proposed the first user attention modelbased framework for video summarization. The scheme used a combination of visual, aural, and linguistic attention models. A non-linear mechanism is then used to fuse all attention models. The attention values of all frames are then used to develop an attention curve, and the crests on the attention curve are selected as the key frames. This framework, even though works well for the shot based key frame extraction, has certain limitations. Firstly, it may produce similar or redundant frames at scene level. Secondly, the relationship between a combination of visual, aural, and linguistic features is difficult to handle. For instance, a background noise or dubbed video footage can have a significant effect on the quality of summaries. Thirdly, the framework is computationally expensive because of employing too many features like object and camera motion, object detection, keyword selection, etc. Peng and Xiaolin [8] used static and dynamic visual attention models to develop a visual attention index with a high priority assigned to dynamic attention model. K-means clustering is used to cluster the frames based on their color histograms. The frames having the highest visual attention index values in each cluster are selected as candidate key frames. The main disadvantage of this scheme is that clustering step does not consider the sequential order of the frames. This means that if color histograms of multiple frames are similar in a few successive shots, they will be clustered together and thus result in missing the potential key frames. Lai and Yi [9] used motion, color, and texture features to build conspicuity maps which are then linearly fused together. A time-constrained clustering algorithm is used to group the similar frames. The frame with the highest saliency value in each cluster is selected as the key frame. The problem with this scheme (and with scheme of [8]) is that the used linear fusion scheme is usually not representative of the complex non-linear human perception mechanism. Ejaz et al. [10] reduced the computational cost of visual attention model by using the temporal gradient-based dynamic visual saliency detection, and discrete cosine transform for static visual attention model has been used. The 
static and dynamic visual attention measures are fused by using a non-linear weighted fusion method. The usage of approximated static and dynamic visual attention models improves efficiency but is likely to effect the quality of summarization.

In all of the above schemes, the motion magnitude based on motion vectors of a frame are utilized for finding out the frames with high level of motion. However, the proposed relative motion intensity feature employs the relative motion magnitude values of two neighboring frames for computation of saliency value. Ma et al. [7] used entropy-based motion consistency measure which takes into account the direction of motion vectors of a single frame. Unlike $\mathrm{Ma}$ et al. [7], our relative motion consistency-based scheme is based on variance and employs the motion variance of the neighboring frame for calculation of variance. In most of the existing schemes, the fusion of features was done linearly which is inadequate. We propose a simple fusion scheme that better reflects the human perception of fusing features.

\section{Methodology}

The proposed framework is based on the notion of visual attention modeling. Attention is a neurobiological concept which denotes the concentration of mental powers upon certain areas by close observation [31]. Visual saliency of a region measures the extent to which it is different from its neighborhood. It is believed that visual saliency highlights the regions which attract visual attention of human beings or which are interesting for humans [31]. Figure 1 shows the main steps of the proposed framework. Each of these steps is described in subsequent sub-sections.

\subsection{Multi-scale color contrast}

The contrast measures the distinctiveness of a region from its environment. The contrast has been widely used for modeling visual attention because the human perception system is known to react to the contrast of visual signals [31]. The contrast value of a pixel is usually computed by calculating the sum of differences of a visual signal with the neighboring pixels. We used the multi-scale contrast [31, 32] for the computation of contrast value at each pixel. The multi-scale contrast is computed on RGB color channels at various scales of Gaussian image pyramid levels.

The contrast value of a color channel $c$ of a pixel $p$ in frame $F$ at a particular scale $l$ of Gaussian pyramid is defined as:

$C_{c}^{l}(F, p)=\sum_{q \in N(p)}\left\|F_{c}^{l}(p)-F_{c}^{l}(q)\right\|^{2}, \mathrm{c}=$ red, green, blue

where $N(p)$ is the $9 \times 9$ neighborhood around pixel $p$, $F_{c}^{l}(p)$ is the value of color channel $c$ at pixel $p$ of frame $F$ at level $l$. The contrast values of each pixel for the three color channels are then added to get the overall contrast value of pixel $p$ at scale $l$.

$$
C^{l}(F, p)=C_{\text {red }}^{l}(F, p)+C_{\text {green }}^{l}(F, p)+C_{\text {blue }}^{l}(F, p)
$$

The resultant contrast value at each pixel is then normalized in the range of $[0,1]$ by dividing the value of each pixel by the maximum contrast value in the frame. The multi-scale contrast value at a pixel is then computed by linear combination of contrasts at various scales of a Gaussian pyramid.

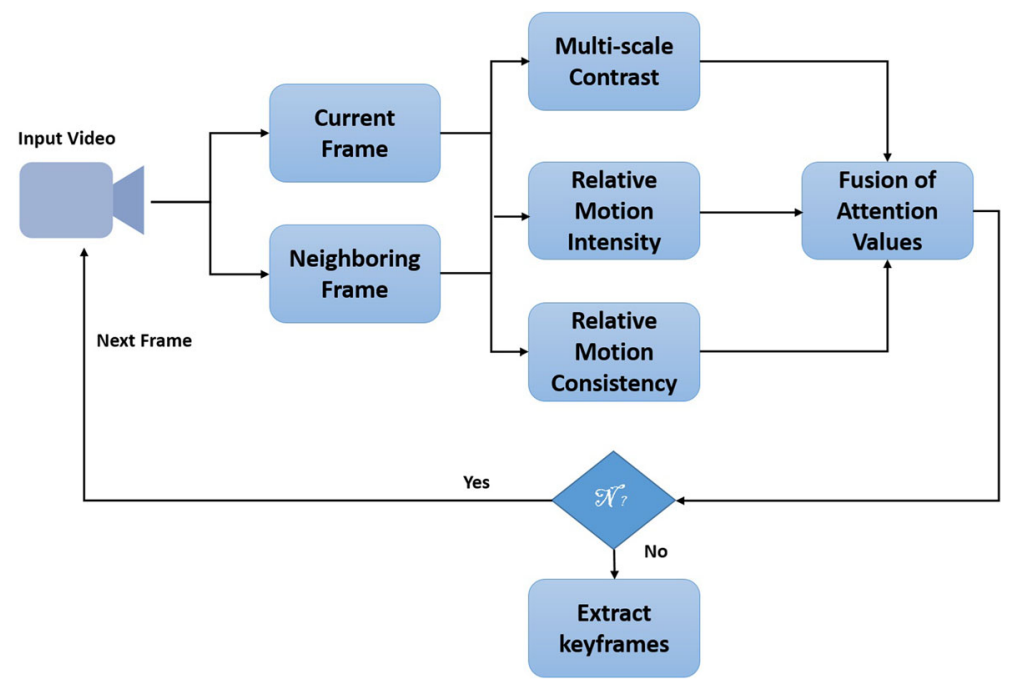

Fig. 1 Framework of the proposed system 


$$
C(F, p)=\sum_{l=1}^{L} C^{l}(F, p)
$$

The number of levels in the Gaussian pyramid $L$ is taken as 6 . The value at each pixel is normalized to the range of $[0,1]$ by dividing each value by the maximum value to obtain a multi-scale contrast saliency map. The average of all non-zero values in the multi-scale color contrast saliency map is then taken as multi-scale color contrast attention value $C(F)$ of frame $F$.

There are multiple benefits of using multi-scale contrast over simple image contrast:

- The computation of contrast at multiple scales helps in locating the salient object in the frame even if the salient objects vary in size,

- It has been asserted in a study by Toet [31] that multi-scale contrast-based visual saliency correlates well with the human perception of visual attention as compared to simple contrast,

- The strength rendered by the usage of multi-scale contrast eliminates the need of using any other static visual saliency model.

A visual comparison of multi-scale contrast and simple contrast saliency maps [7] is shown in Fig. 2. It is clear that multi-scale contrast outperforms simple contrast by clearly identifying only the salient object in the scene.

\subsection{Dynamic features}

In static images, the saliency maps are indexed on image regions, whereas in dynamic scenes, humans focus both on objects and their motion [31]. Moreover, the relative motion among objects is also important in user attention modeling. Therefore, in videos, motion is an important factor in building human attention model. Because of this importance of motion, two different descriptors based on relative motion strength and relative motion direction have been proposed for building saliency maps. For computation of these maps, first, the motion vector field $(\mathrm{M})$ is computed using the Lucas Kanade Algorithm [33]. The resultant motion vectors are then used to compute the two descriptors called relative motion intensity and relative motion consistency.

\subsubsection{Relative motion intensity}

In most of the previous works, the motion intensity was directly used as a visual attention feature. If $M_{x}(p)$ and $M_{y}(p)$ represent the $x$ and $y$ component of the motion vector of current frame $F$ with reference to previous frame, the motion intensity at pixel $p$ is given as:

$$
I(F, p)=\sqrt{M_{x}^{2}(p)+M_{y}^{2}(p)}
$$

In this paper, instead of using motion intensity directly, the notion of relative motion intensity has been introduced. For videos, the relative motion intensity is expected to be more relevant than the absolute motion intensity.

For the calculation of relative motion intensity-based attention measure, the motion intensity of a frame is computed using Eq. (4). Next, the frame is divided into non-overlapping blocks of size $9 \times 9$. The human beings tend to focus more towards center of an image. Therefore, a weight $\alpha$ is defined in such a way that the blocks near to the center of the frame are assigned higher weight as compared to the blocks that are far from the center of the frame. For this purpose, the distance of each block with the center of the frame is computed. This distance measure is used to define weight $\alpha$ in such a way that the weight value is higher if the distance between a given block center with the center of the frame is less and vice versa. If $d_{\max }$ represents the maximum possible Euclidean distance between a block and center of image and $d_{i}$ represents the Euclidean distance of block $i$ from the center of image, then the weight $\alpha_{i}$ for block $i$ is defined as:

$$
\alpha_{i}=1-\frac{d_{i}}{d_{\max }}
$$

The average motion magnitude of each block $i$ denoted by $I_{i}(F, p)$ is then found by averaging the motion intensity of each pixel in the block. The motion intensity of each
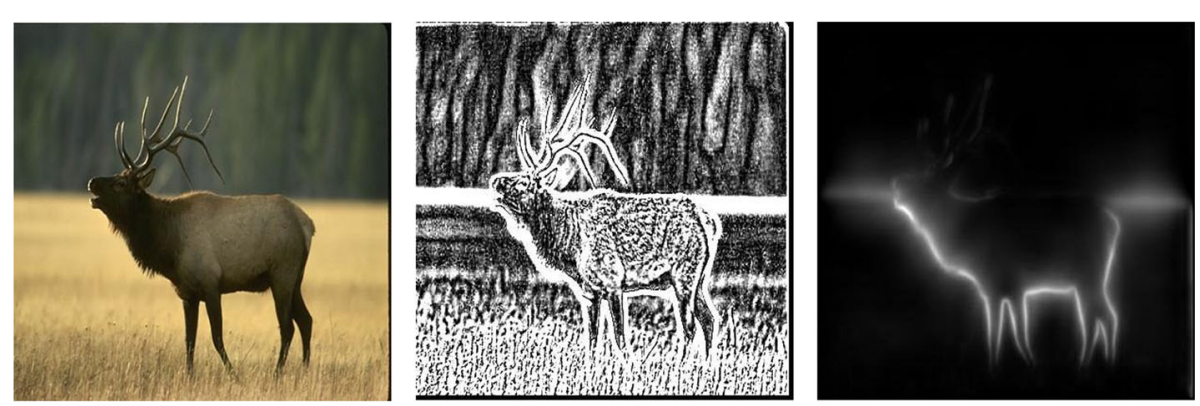

Fig. 2 Comparison of simple color contrast and multi-scale color contrast. From left to right: actual image, simple color contrast, and multi-scale contrast 
block $i$ is multiplied by weight $\alpha_{i}$. The weighted motion intensity of each block is added to get the weighted motion intensity of frame $F$.

The weighted average motion intensity value is then computed for each frame in the video. If there are $n$ frames in the video and $I_{F j}$ represents the weighted average intensity value of $j$ th frame, $I_{\min }$ and $I_{\max }$ are the minimum and maximum motion intensity values, then relative motion intensity of frame $R I\left(F_{j}\right)$ is found as:

$$
R I\left(F_{j}\right)=\frac{I_{F j}-I_{\min }}{I_{\max }-I_{\min }}
$$

The relative motion intensity values are now in the range of $[0,1]$ whereby a value closer to 1 represents a high salient relative motion and a value closer to 0 means less salient motion.

\subsubsection{Relative motion consistency}

Along with the motion magnitude, an important metric in the motion field is the orientation or direction of the motion vectors. If $M_{x}(p)$ and $M_{y}(p)$ represent the $x$ and $y$ components of the motion vector of current frame $F$ with reference to previous frame, the orientation value at pixel $p$ is given as:

$$
O(F, p)=\arctan \left(\frac{M_{y}(p)}{M_{x}(p)}\right)
$$

The consistent values of orientation generally represent the saliency intensive moving objects. A set of inconsistent orientation values, on the other hand, may refer to object boundaries or random motions.

In order to compute the relative motion consistency measure of a frame, the orientation value of each pixel in the frame is computed using Eq. (7). Like, Section 3.2.1, the frame is divided into non-overlapping blocks of size $9 \times 9$. The weight values $\alpha_{i}$ of a block $i$ relative to the center of the image is also defined in same way as in Section 3.2.1. Next, the variance of each block $i$ denoted by $\sigma_{i}^{2}$ is computed which is a measure of variation of motion orientations in the corresponding block. The variance value of each block $i$ is then multiplied by the corresponding weight $\alpha_{i}$. The variances of each block in the frame is then summed up to get the motion variance measure of each frame.

The weighted average motion variance value is then computed for each frame in the video. If there are $n$ frames in the video and $\sigma_{F j}^{2}$ represents the weighted average variance value of $j$ th frame, $\sigma_{\min }^{2}$ and $\sigma_{\max }^{2}$ are the minimum and maximum motion variance values, then the relative motion consistency value of frame $F_{j}$ denoted by $R O\left(F_{j}\right)$ is found as:

$$
R O\left(F_{j}\right)=1-\frac{\sigma_{F j}^{2}-\sigma_{\min }^{2}}{\sigma_{\max }^{2}-\sigma_{\min }^{2}}
$$

In Eq. (8), the variance values are subtracted from 1 to convert relative variance values into relative consistency values. The resultant values are again in the range of $[0,1]$. The values closer to 1 indicates relatively higher consistent motion, and the values closer to 0 indicates relatively lower consistent motion.

\subsubsection{Benefits of relative motion measures}

The relative motion intensity, unlike absolute motion intensity, determines the amount of motion relative to the rest of the frames. The studies of human visual systems indicate that the human tend to focus on that parts of the video which reflect more change in terms of motion. The proposed relative motion intensity and relative motion consistency measure the change in magnitude and direction in a frame relative to other frames in the video. In this way, those frames will get the higher visual attention values where the change in higher in comparison with other frames. On the other hand, the usage of absolute measures for magnitude and direction will merely capture the amount and direction of motion in the frame irrespective of the amount and direction of motion in other frames and thus will not effectively reflect the properties of human visual system.

Moreover, the proposed methods of relative motion take into account the location of a pixel value relative to the center of the image. Since, human beings usually focus on the center of an image, so the motion values are adjusted in a way such that the pixels closer to center get a high bias as compared to the pixels way from the center. The proposed scheme assigns this bias on block level instead of applying on pixel level in order to save the computational time.

\subsection{Fusion of features}

Most of the techniques in literature use a linear fusion scheme for combining various metrics of visual saliency in which all the values are assigned a weight and then added [31]. However, it is believed that the visual section of the human brain uses non-linear processing systems for the tasks of pattern recognition and classification [34]. Moreover, this type of linear combination may not reflect all the information contained by the attention values of the attention components [34]. Consider, for example, a pair of two attention values $(1,0)$ where 1 is the static attention value and 0 is the dynamic attention attention value. The values indicate that a frame is highly significant from static perspective but probably because of lack of motion the value of dynamic attention value is 0 . Nevertheless, the frame is still considered to be highly relevant from the perspective of visual attention. A linear fusion scheme simply averages the two values. But the resultant value of 0.5 does not reflect the importance of the frame from static perspective. Another fusion possibility 
is to select the maximum value of the two attention values. However, the maximum function does not differentiate between values $(1,0),(1,0.9),(1,0.8)$, etc. where the resultant value will always be 1 and ignores the second attention value altogether.

In this context, a fusion scheme with the following properties is needed:

- For two high attention values, the resultant fusion value must be high.

- If one attention value is high and other is low, the resultant fusion value must still be relatively high.

- If both attention values are low, the resultant fusion value must be low.

- The fusion function must be an an increasing function. For instance, if one attention value is 1 and other attention value vary from 0 to 1 , then the fusion values must be increasing.

A simple novel fusion mechanism is proposed that fulfills all of the abovementioned properties. Consider $A_{1}$ and $A_{2}$ to be two attention values to be fused. The value of a constant $c_{1}$ is then defined as per following criteria:

$$
c_{1}= \begin{cases}0.2 & \text { if } \max \left(A_{1}, A_{2}\right) \geq \lambda \\ \lambda & \text { otherwise }\end{cases}
$$

The threshold $\lambda$ is defined to be 0.7. Another constant $c_{2}$ is set to the value 0.2 . The two attention values are then combined to get an intermediate fused value $F^{\prime}$ :

$$
F^{\prime}=\frac{\left[1+\max \left(A_{1}, A_{2}\right)-c_{1}\right]+\left[\min \left(A_{1}, A_{2}\right)-c_{2}\right]}{2}
$$

Using Eq. (10), the maximum possible value is achieved when one of the attention values is 1 and the minimum attention value is achieved when one of the values is 0 . Using Eq. (10), the maximum and minimum possible values $F_{\max }^{\prime}$ and $F_{\min }^{\prime}$ are found as:

$$
\begin{aligned}
& F_{\max }^{\prime}=\frac{\left[2-c_{1}\right]+\left[\min \left(A_{1}, A_{2}\right)-c_{2}\right]}{2} \\
& F_{\text {min }}^{\prime}=\frac{1+\max \left(A_{1}, A_{2}\right)-c_{1}-c_{2}}{2}
\end{aligned}
$$

Finally, the fused attention value $F$ is obtained:

$$
F=\left(\max \left(A_{1}, A_{2}\right)-F_{\min }^{\prime}\right) \times\left(\frac{F^{\prime}-F_{\min }^{\prime}}{F_{\max }^{\prime}-F_{\min }^{\prime}}\right)+F_{\min }^{\prime}
$$

The threshold $\lambda$ determines the attention value that is considered to be high. Setting $\lambda=0.7$ suggests that an attention value that is greater than or equal to 0.7 is considered high. The constants $c_{1}$ and $c_{2}$ are controlling parameters. Equation (9) suggests that if one of the attention values exceeds the criteria of being high then the penalty control parameter $c_{1}$ is set to be low and vice versa. The second controlling parameter $c_{2}$ is fixed to a low value. Equation (10) is averaging the attention values by boosting the maximum value by adding 1 and then penalizing the maximum and minimum value by subtracting $c_{1}$ and $c_{2}$ respectively. The choice of parameters $c_{1}$ and $c_{2}$ makes sure that if at least one of the attention values is higher then the penalty to the higher value is less. However, if both values are lower, then the first value also gets a higher penalty. Equations (11) and (12) simply computes the minimum and maximum possible values of Eq. (10). Equation (13) simply scales the fusion value obtained through Eq. (10) in the range [0,1]. The value of $\lambda$ can be changed to set to a value that is considered to be high. The values of controlling parameters $c_{1}$ and $c_{2}$ can be changed to control the level of penalty to low and high attention values. The values of parameters given above are selected experimentally.

Table 1 displays the results of fusion values for combination of two attention values in the range of $[0,1]$ with a step size of 0.1. As discussed, the fusion scheme must generate a higher resultant value if one of the attention value is on the higher side. Moreover, the magnitude of the attention value must also be reflected in the fused value. The results indicate that the fusion scheme fulfills all the properties of an ideal fusion scheme of attention values.

\subsection{Key frame selection}

The range of the attention values for the complete video is increased by stretching the range of attention values to span the entire range of values from 0 to 1 . This range stretching step assists in highlighting the differences between attention values. If the minimum and maximum values in the attention curve are denoted by $A_{\min }$ and $A_{\max }$, then each value $A_{\text {in }}$ in the attention curve is scaled to $A_{\text {out }}$ as:

$$
A_{\text {out }}=\frac{A_{\text {in }}-A_{\text {min }}}{A_{\max }-A_{\min }}
$$

The key frame selection module is flexible and can be changed as per application. In some applications, the number of key frames is fixed beforehand by the users. In this case, there are three possibilities. The first possibility is that the number of desired key frames is equal to the number of shots in the video. In this case, the frame with the highest attention value in each shot is selected as key frame. The second possibility is that the number of desired key frames is less than the total number of shots in the video. In this case, one candidate key frame is selected from each shot and the candidate frames with lower attention values are discarded. If the desired number of key frames is more than the number of shots, then the shots with more variation are assigned more key frames [9]. The 
Table 1 Fusion results for sample attention values

\begin{tabular}{llllllllllll}
\hline$A_{1} / A_{2}$ & 1 & 0.9 & 0.8 & 0.7 & 0.6 & 0.5 & 0.4 & 0.3 & 0.2 & 0.1 & 0 \\
\hline 1 & 1 & 0.98 & 0.96 & 0.94 & 0.92 & 0.9 & 0.88 & 0.86 & 0.84 & 0.82 & 0.8 \\
0.9 & 0.98 & 0.885 & 0.87 & 0.855 & 0.84 & 0.825 & 0.81 & 0.795 & 0.78 & 0.765 & 0.75 \\
0.8 & 0.96 & 0.87 & 0.78 & 0.77 & 0.76 & 0.75 & 0.74 & 0.73 & 0.72 & 0.71 & 0.7 \\
0.7 & 0.94 & 0.855 & 0.77 & 0.685 & 0.68 & 0.675 & 0.67 & 0.665 & 0.66 & 0.655 & 0.65 \\
0.6 & 0.92 & 0.84 & 0.76 & 0.68 & 0.5 & 0.475 & 0.45 & 0.425 & 0.4 & 0.375 & 0.35 \\
0.5 & 0.9 & 0.825 & 0.75 & 0.675 & 0.475 & 0.4 & 0.38 & 0.36 & 0.34 & 0.32 & 0.3 \\
0.4 & 0.88 & 0.81 & 0.74 & 0.67 & 0.45 & 0.38 & 0.31 & 0.295 & 0.28 & 0.265 & 0.25 \\
0.3 & 0.86 & 0.795 & 0.73 & 0.665 & 0.425 & 0.36 & 0.295 & 0.23 & 0.22 & 0.21 & 0.2 \\
0.2 & 0.84 & 0.78 & 0.72 & 0.66 & 0.4 & 0.34 & 0.28 & 0.22 & 0.16 & 0.155 & 0.15 \\
0.1 & 0.82 & 0.765 & 0.71 & 0.655 & 0.375 & 0.32 & 0.265 & 0.21 & 0.155 & 0.1 & 0.1 \\
0 & 0.8 & 0.75 & 0.7 & 0.65 & 0.35 & 0.3 & 0.25 & 0.2 & 0.15 & 0.1 & 0.05 \\
\hline
\end{tabular}

variation of each shot is measured by computing the variance of fused attention values of the frames in the shot. If the variation in a shot is higher, it must be assigned more key frames and vice versa. The number of key frames assigned to a particular shot is then defined as:

$$
K_{s}=\max \left(n_{K} \times \frac{v_{s}}{\sum_{i=1}^{T_{s}} v_{i}}, 1\right)
$$

$v_{s}$ is the variance of attention values in a shot $s, n_{K}$ is the total number of desired key frames, and $T_{s}$ is the number of shots in the video. If the desired number of key frames is unknown, then the frame with the highest attention value is selected as the key frame from each shot.

\section{Experiment results and discussion}

In order to evaluate the efficacy of the proposed method, three different groups of experiments were performed. The details of these experiments are described in the subsequent sections.

\subsection{Benefits of proposed visual attention mechanism}

In this sub-section, the benefits of the proposed scheme have been demonstrated based on the choice of key frames in single shots of the two videos downloaded from the Open Video project www.open-video.org.

Firstly, we present the results of the proposed technique on frames 532 to 548 from the second shot of the video hcil2000_01.mpeg. The shot consists of a person standing and talking in front of the trees with very little motion. A subtitle, showing an introduction of the person in the scene starts appearing in Frame 545. There is no significant motion in the scene apart from the movement of the person's body parts. The maximum attention value is found at frame 548, and thus, it is selected as the key frame. Figure 3 shows the key frames selected by a clustering-based algorithm [14], visual attentionbased model [9], and the proposed method. The key frame selected by [14] does not include the subtitle. In the key frame selected by [9], it can be seen that even though the subtitle starts appearing in the frame, it is not legible. The key frame selected by our scheme matches the human perception better than the key frame extracted by [9].

Secondly, the technique was tested on the fifth shot of the video ucomp03_0 6_m1.mpeg which ranges from the frames 484 to 555 . This shot shows a tennis player striking the ball and then standing and receiving applause from the
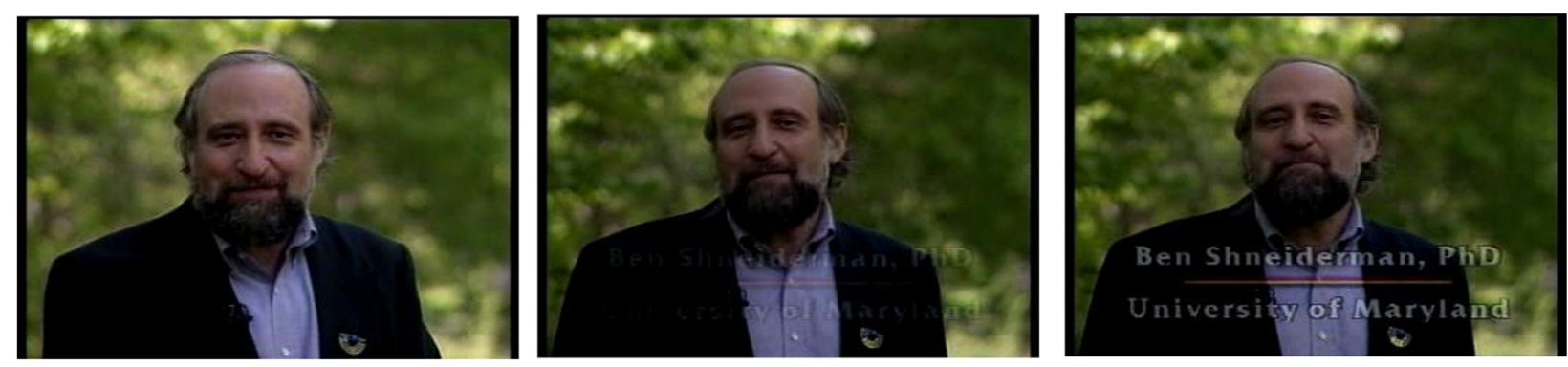

Fig. 3 Key frames extracted by [9, 14] and our scheme on the video hci2000_01.mpg. From left to right: a key frame extracted by [14], b key frame extracted by [9], and c key frame extracted by proposed scheme 

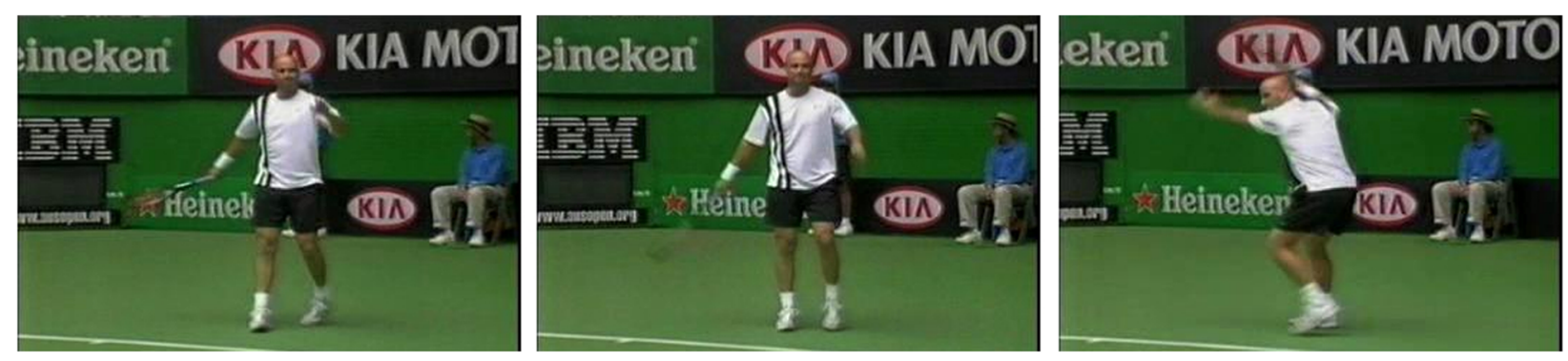

Fig. 4 Key frames extracted by [14] and [9] and our scheme on the video ucomp03_06_m1.mpeg. From left to right: a key frame extracted by [14], b key frame extracted by [9], c key frame extracted by the proposed scheme

audience. The shot is characterized by a large amount of camera and object motions. The highest attention value is present at frame 486 which is thus the key frame selected by our scheme.Lai and Yi [9] selects frame 525 as the key frame. Figure 4 shows the key frames extracted by [14] and [9] and the proposed technique. The key frames extracted by [14] and [9] fail to show the stroke of the tennis player. The key frame extracted by our scheme is thus more interesting and representative of the shot.

\subsection{Comparison with other techniques}

In this section, the results of our scheme are compared with some of the popular non-visual attention-based techniques. For this purpose, the experiments are conducted based on 20 videos selected from the Open Video Project. The videos belong to different genres including historical, education, and documentary. These videos were part of the data set used by many authors [12-14] in the evaluation of their video summarization algorithms. All the videos are in mpeg format. The information about the data set videos is presented in Table 2. For the evaluation data set of Table 2, the proposed scheme has been compared with four other schemes for key frame extraction: OV [11], DT [12], STIMO [13], and VSUMM [14].

In the evaluation scheme, the key frames are manually selected by three human users for a particular video and then compared with the key frames extracted by a particular technique. The number of matched and unmatched key frames is then calculated. The two frames are considered matched if they are conveying same semantic message. In this way, the key frames selected by the user act as ground truth data. The comparison between ground truth and key frames generated by a technique is used to define the following terms: true positive, a frame selected as key frame by both human user and the technique; false positive, a frame selected as key frame by technique but not by human user; and false negative, a frame selected as key frame by human user but not by technique.

The number of true positive, false positive, and false negative frames is used to reflect the quality of the summaries in terms of popular pattern recognition metrics Recall, Precision, and F-measure. In the current context, the Recall is defined as the probability that a relevant key frame is selected by the technique. Precision is defined as the probability that an extracted key frame is relevant. Usually, there is a tradeoff between Recall and Precision values where one value decreases with the increase in the other one. The F-measure combines Precision and Recall as one measure.

If the numbers of true positive, false positive, and false negative frames are denoted by $T \mathrm{p}, F \mathrm{p}$, and $F \mathrm{n}$ respectively, Recall and Precision are defined as:

Table 2 Details of test videos

\begin{tabular}{lll}
\hline No. & Video name & $\begin{array}{l}\text { No. of } \\
\text { frames }\end{array}$ \\
\hline 1 & Wetlands Regained, segment 03 of 8 & 3562 \\
2 & Technology at Home: A Digital Personal Scale & 3346 \\
3 & Introduction to HCIL 2000 reports & 2454 \\
4 & Ocean floor Legacy, segment 05 of 14 & 4665 \\
5 & The Great Web of Water, segment 01 & 3279 \\
6 & The Great Web of Water, segment 02 & 2118 \\
7 & The Great Web of Water, segment 07 & 1745 \\
8 & A New Horizon, segment 01 & 1806 \\
9 & A New Horizon, segment 02 & 1797 \\
10 & A New Horizon, segment 06 & 1944 \\
11 & A New Horizon, segment 08 & 1815 \\
12 & Exotic Terrene, segment 04 & 4797 \\
13 & The Future of Energy Gases, segment 05 & 3615 \\
14 & The Future of Energy Gases, segment 09 & 1884 \\
15 & Ocean floor Legacy, segment 01 & 1740 \\
16 & Ocean floor Legacy, segment 02 & 2325 \\
17 & Ocean floor Legacy, segment 09 & 2106 \\
18 & Hurricane Force—A Coastal Perspective, segment 03 & 2310 \\
19 & Drift Ice as a Geologic Agent, segment 05 & 2187 \\
20 & Drift Ice as a Geologic Agent, segment 10 & 1407 \\
\hline
\end{tabular}


Table 3 Mean Recall, Precision, and F-measure achieved by different techniques

\begin{tabular}{llllll}
\hline & OV & DT & STIMO & VSUMM & Proposed \\
\hline Recall & 0.65 & 0.56 & 0.70 & 0.73 & 0.88 \\
Precision & 0.67 & 0.67 & 0.64 & 0.71 & 0.82 \\
Recall & 0.64 & 0.61 & 0.65 & 0.71 & 0.85 \\
\hline
\end{tabular}

$$
\begin{aligned}
& \text { Recall }=\frac{T_{\mathrm{p}}}{T_{\mathrm{p}}+F_{\mathrm{n}}} \\
& \text { Precision }=\frac{T_{\mathrm{p}}}{T_{\mathrm{p}}+F_{\mathrm{p}}}
\end{aligned}
$$

The Recall and Precision obtained by matching with summaries of three users are then averaged to obtain Precision and Recall values for a video. The F-measure is then defined as:

$$
F=2 \times\left(\frac{\text { Recall } \times \text { Precision }}{\text { Recall }+ \text { Precision }}\right)
$$

The ground truth data (user summaries) for the data set of Table 1 is taken from the open access database provided by the authors of [14]. The summaries for our technique have been generated by selecting one key frame from each shot. The average values of Recall, Precision, and F-measure for all the techniques under consideration are shown in Table 3 . The proposed methodology is clearly superior to the rest of the techniques based on these results.

The Recall, Precision, and F-measure values for each video in the evaluation data set are shown in Fig. 5. It can be easily observed that the proposed technique consistently achieve the highest values for Recall and Precision for all the videos. There are however exceptions whereby other schemes attain high values for one of the parameters. A high value for one of the parameters is generally not sufficient. The Precision measures the ability of a scheme to retrieve the most precise results. A high value of Precision means better relevance. However, a high value of Precision can be achieved by selecting very few key frames. For instance, for video 5, DT achieves a Precision value of 1 by selecting only one key frame. The Recall value measures the ability to retrieve as many as possible relevant key frames. In this way, the Recall value complements the Precision value. Therefore, a good scheme is one having high values for both the Recall and Precision. Thus, the Recall value of DT for video 5 is sufficiently low. The proposed scheme however has the highest value of Recall for this video and a reasonably high value for Precision. Moreover, the F-measure of our scheme is 0.79 whereas for DT it is 0.77 . Table 3 clearly indicates that the proposed scheme obtains the highest value for F-measure and thus is more efficient and close to human perception than that of the other schemes.

\subsection{Time complexity of the proposed system}

Assume that there are a total of $T$ number of frames in the image with each one of them having resolution $N \times N$.

The time complexity for computing the relative motion intensity value for one frame is the sum of (i) time taken for computation of optical flow which is $O\left(N^{2}\right)$ using Lucas Kanade algorithm by setting the number of warp parameters to 2, (ii) finding the distance of each of the $N / 2$ block with the center of the frame which is $O(N)$, (iii) finding and applying the value of $\alpha$ which is again an operation of $O(N)$, (iv) finding the average intensity value which involves finding average value of each pixel in the frame and thus is $O\left(N^{2}\right)$, and (v) finding the relative motion intensity which involves finding the relative intensity of a frame in comparison with all frames of the image and thus has a time complexity of $O(T)$. Thus, the overall time complexity of finding relative motion intensity is $O\left(N^{2}+T\right)$.

Similarly, the time complexity of finding relative orientation consistency is $O\left(N^{2}+T\right)$. The time complexity of fusion of features is $O(1)$ as there are only three features to be combined. The time complexity of key frame selection is $O(T)$. Thus, the overall time complexity of the system is $O\left(N^{2}+T\right)+O\left(N^{2}+T\right)+O(T)=O\left(N^{2}+T\right)$.
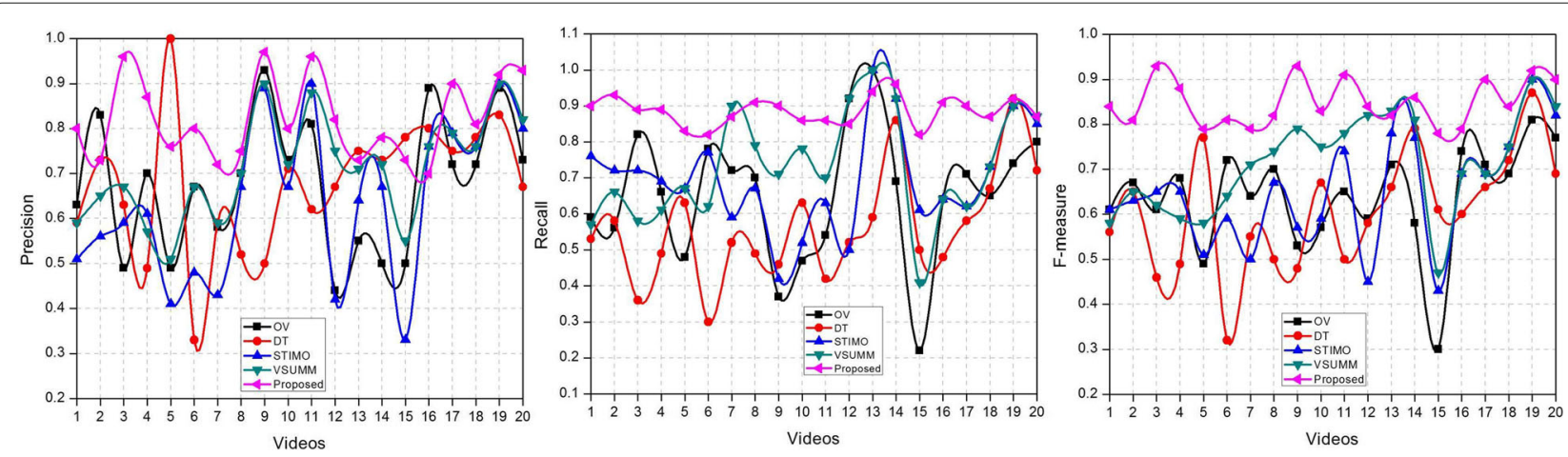

Fig. 5 Recall (R), Precision (P), and F-measure (F) achieved by different techniques on video data set of Table 2 


\section{Conclusions}

In this paper, a visual attention-based framework for extracting key frames from the videos is presented. The proposed saliency-based visual attention model effectively bridges the semantic gap between low level features and human perception without actual human intervention. The usage of multi-scale color contrast provides an excellent approximation of the interested object in a video frame. The proposed relative motion intensity and relative orientation consistency are effective measures of motion-based saliency. The used fusion scheme combines the benefits of previously used schemes by providing a non-linear mechanism along with weighted motion priority mechanism. The used visual attention clues and fusion mechanism render a more complete and consistent framework. The framework allows the users to control the density of key frames and works at both shot and scene level. The experimental results on a number of criteria demonstrate that the proposed framework extracts more semantically meaningful key frames as compared to the techniques to which it is compared. The time complexity of the algorithm is the major issue which may hinder the implementation in some scenarios. This can be improved by using more efficient algorithm for computing optical flow or by using an efficient motion estimation scheme.

\section{Funding}

This work was supported by the National Research Foundation of Korea (NRF) grant funded by the Korea Government (MSIP) (No. 2016R1A2B4011712).

\section{Authors' contributions}

NE, SWB, HC, and IM conceived and discussed the project. IM and NE developed and discussed the algorithms. SWB and $\mathrm{HC}$ wrote the publication and provided the data for testing the algorithms. HM assisted in testing and revising the paper apart from doing time complexity analysis. All authors have read and approved the final manuscript.

\section{Competing interests}

The authors declare that they have no competing interests.

\section{Publisher's Note}

Springer Nature remains neutral with regard to jurisdictional claims in published maps and institutional affiliations.

\section{Author details}

${ }^{1}$ Department of Computer Science, Iqra University, Islamabad, Pakistan.

${ }^{2}$ Department of Software, Sejong University, Seoul, South Korea. ${ }^{3}$ Department of Computer Science, National University of Computer and Emerging Sciences, Islamabad, Pakistan. ${ }^{4}$ Department of Industrial Security, College of Business and Economics, Chung-Ang University, Seoul, South Korea.

Received: 12 January 2018 Accepted: 21 May 2018

Published online: 05 June 2018

\section{References}

1. BT Truong, S Venkatesh, Video abstraction: a systematic review and classification, (2007), pp. 1-37

2. AG Money, H Agius, Video summarisation: a conceptual framework and survey of the state of the art. J. Vis. Commun. Image Represent. 19, 121-143 (2008)
3. X Song, L Sun, J Lei, D Tao, G Yuan, M Song, Event-based large scale surveillance video summarization. Neurocomputing. 187, 66-74 (2016) Recent Developments on Deep Big Vision

4. T Itazuri, T Fukusato, S Yamaguchi, S Morishima, in Hawaii Convention Center in Honolulu. Court-based volleyball video summarization focusing on rally scene, (Hawaii, 2017), pp. 179-186

5. N Ejaz, TB Tariq, SW Baik, Adaptive key frame extraction for video summarization using an aggregation mechanism. J. Vis. Commun. Image Represent. 23, 1031-1040 (2012)

6. AC Hernandez, MC Hernandez, FG Ugalde, MN Miyatake, HP Meana, A fast and effective method for static video summarization on compressed domain. IEEE Lat. Am. Trans. 14, 4554-4559 (2016)

7. YF Ma, LLu XS Hua, HJ Zhang, A generic framework of user attention model and its application in video summarization. IEEE Trans. Multimedia. 7, 907-919 (2005)

8. J Peng, Q Xiaolin, Keyframe-based video summary using visual attention clues. IEEE MultiMedia. 17, 64-73 (2010)

9. J-L Lai, Y Yi, Key frame extraction based on visual attention model. J. Vis. Commun. Image Represent. 23, 114-125 (2012)

10. N Ejaz, I Mehmood, SW Baik, Efficient visual attention based framework for extracting key frames from videos.Signal Process. Image Commun. 28 34-44 (2013)

11. D DeMenthon, V Kobla, D Doermann, in Proceedings of the Sixth ACM International Conference on Multimedia. MULTIMEDIA '98. Video summarization by curve simplification (ACM, New York, 1998), pp. $211-218$

12. P Mundur, Y Rao, Y Yesha, Keyframe-based video summarization using Delaunay clustering. Int. J. Digit. Libr. 6, 219-232 (2006)

13. M Furini, F Geraci, M Montangero, M Pellegrini, Stimo: Still and moving video storyboard for the web scenario. Multimedia Tools Appl. 46, 47 (2009)

14. SEF de Avila, APB Lopes, A da Luz, A de Albuquerque Arajo, Vsumm: A mechanism designed to produce static video summaries and a novel evaluation method. Pattern Recogn. Lett. 32, 56-68 (2011). Image Processing, Computer Vision and Pattern Recognition in Latin America

15. D Potapov, M Douze, Z Harchaoui, C Schmid, Category-Specific Video Summarization. (Springer International Publisher, Switzerland, 2016), pp. 540-555

16. M Sun, A Farhadi, S Seitz, in IEEE, Computer vison and pattern recognition, Hynes Convention Center in Boston. Ranking Domain-Specific Highlights by Analyzing Edited Videos (Springer International Publishing, Massachusetts, 2016), pp. 787-802

17. M Gygli, H Grabner, LV Gool, in IEEE, Computer vison and pattern recognition, Hynes Convention Center in Boston. Video summarization by learning submodular mixtures of objectives, (Massachusetts, 2015), pp. 3090-3098

18. KZhang, WL Chao, F Sha, K Grauman, in IEEE, 2015 International Conference on Computer Vision, Dates: Dec 11, 2015 Ü Dec 18, 2015. Summary transfer: exemplar-based subset selection for video summarization, (Araucano Park, Las Condes, Chile, 2016), pp. 1059-1067

19. B Xiong, G Kim, L Sigal, in 2013 IEEE Conference on Computer Vision and Pattern Recognition, Hynes Convention Center in Boston. Storyline representation of egocentric videos with an applications to story-based search, (Massachusetts, 2015), pp. 4525-4533

20. Z Lu, K Grauman, in IEEE, 201422 nd International Conference on Pattern Recognition. Story-driven summarization for egocentric video, (Stockholm, Sweeden, 2013), pp. 2714-2721

21. R Panda, SK Kuanar, AS Chowdhury, in 2013 IEEE Conference on Computer Vision and Pattern Recognition, Hynes Convention Center in Boston. Scalable video summarization using skeleton graph and random walk, (Massachusetts, 2014), pp. 3481-3486

22. WS Chu, Y Song, A Jaimes, in 2012 IEEE Conference on Computer Vision and Pattern Recognition, Hynes Convention Center in Boston. Video co-summarization: video summarization by visual co-occurrence, (Massachusetts, 2015), pp. 3584-3592

23. S Feng, Z Lei, D Yi, SZ Li, in 2015 IEEE Conference on Computer Vision and Pattern Recognition, Hynes Convention Center in Boston. Online content-aware video condensation, (Massachusetts, 2012), pp. 2082-2087

24. F Dornaika, IK Aldine, Decremental sparse modeling representative selection for prototype selection. Pattern Recognit. 48, 3714-3727 (2015)

25. E Elhamifar, G Sapiro, R Vidal, in IEEE, Computer vison and pattern recognition, Hynes Convention Center in Boston. See all by looking at a few: sparse modeling for finding representative objects, (Massachusetts, 2012), pp. 1600-1607 
26. J Meng, H Wang, J Yuan, YP Tan, in IEEE, Computer vison and pattern recognition, Hynes Convention Center in Boston. From keyframes to key objects: video summarization by representative object proposal selection, (Massachusetts, 2016), pp. 1039-1048

27. B Zhao, EP Xing, in IEEE Conference on Computer Vision and Pattern Recognition. Quasi real-time summarization for consumer videos (Elsevier International Publisher, Switzerland, 2014), pp. 2513-2520

28. H-C Lee, S-D Kim, Iterative key frame selection in the rate-constraint environment. Signal Process. Image Commun. 18, 1-15 (2003)

29. T Liu, X Zhang, J Feng, K-T Lo, Shot reconstruction degree: a novel criterion for key frame selection. Pattern Recogn. Lett. 25, 1451-1457 (2004)

30. M Cooper, J Foote, in IEEE International Conference on Multimedia and Expo. Discriminative techniques for keyframe selection (Elsevier International Publisher, Switzerland, 2005), p. 4

31. A Toet, Computational versus psychophysical bottom-up image saliency: a comparative evaluation study. IEEE Trans. Pattern. Anal. Mach. Intell. 33, 2131-2146 (2011)

32. T Liu, Z Yuan, J Sun, J Wang, N Zheng, X Tang, HY Shum, Learning to detect a salient object. IEEE Trans. Pattern. Anal. Mach. Intell. 33, 353-367 (2011)

33. BD Lucas, T Kanade, in Proceedings of the 7 th International Joint Conference on Artificial Intelligence - Volume 2, IJCAl'81. An iterative image registration technique with an application to stereo vision (Morgan Kaufmann Publishers Inc, San Francisco, 1981), pp. 674-679

34. D Sánchez, J Chamorro-Martínez, M Vila, Modelling subjectivity in visual perception of orientation for image retrieval. Inf. Process. Manag. 39, 251-266 (2003)

\section{Submit your manuscript to a SpringerOpen ${ }^{\circ}$ journal and benefit from:}

- Convenient online submission

- Rigorous peer review

- Open access: articles freely available online

- High visibility within the field

- Retaining the copyright to your article

Submit your next manuscript at $>$ springeropen.com 